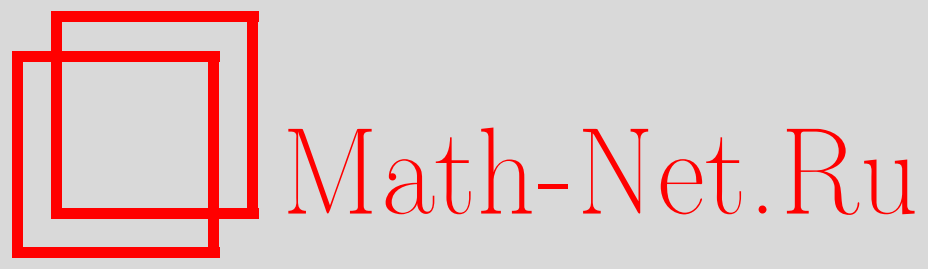

М. Т. Караев, Применение теории банаховых алгебр к формуле сложения спектральных кратностей семейств операторов, Функи. анализ и его прил., 2007, том 41, выпуск 1, 93-95

DOI: https://doi.org/10.4213/faa2855

Использование Общероссийского математического портала Math$\mathrm{Net.Ru}$ подразумевает, что вы прочитали и согласны с пользовательским соглашением

http://www. mathnet.ru/rus/agreement

Параметры загрузки:

IP : 54.84 .234 .179

26 апреля 2023 г., 14:46:30

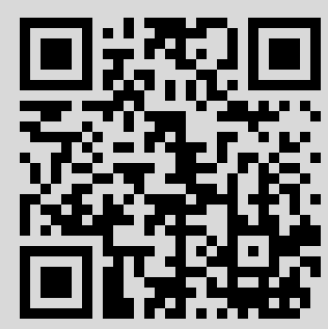




\title{
Применение теории банаховых алгебр к формуле сложения спектральных кратностей семейств операторов
}

\author{
(C) 2007. M. T. KAPAEB
}

1. Введение. Обозначим через $\mathscr{B}(X, Y)$ пространство ограниченных линейных операторов из банахова пространства $X$ в банахово пространство $Y$; в случае когда $X=Y$, мы будем писать просто $\mathscr{B}(X)$. Пусть $\mathscr{A}-$ банахова алгебра, а $\pi: \mathscr{A} \rightarrow \mathscr{B}(X)$ - ее представление. Если в алгебре $\mathscr{A}$ есть единица $e$, мы требуем, чтобы $\pi(e)=I$. Определим для элемента $a \in \mathscr{A}$ оператор $L_{a} \in \mathscr{B}(\mathscr{A})$ условием $L_{a}(x)=a x$. Отображение $L: \mathscr{A} \rightarrow \mathscr{B}(\mathscr{A}), L(a)=L_{a}$, является алгебраическим гомоморфизмом и называется регулярным представлением алгебры $\mathscr{A}$.

Напомним, что множество $M \subset X$ называется циклическим для семейства операторов $\mathscr{T}$ на банаховом пространстве $X$, если наименьшее $\mathscr{T}$-инвариантное подпространство пространства $X$, содержащее $M$, совпадает с $X$. Минимальная из мощностей циклических множеств для семейства $\mathscr{T}$ называется его спектральной кратностью и обозначается через $\mu(\mathscr{T})$. Представление $\pi$ алгебры $\mathscr{A}$ называется циклическим, если существует такой вектор $x \in X$, что множество $\{\pi(a) x: a \in \mathscr{A}\}$ плотно в $X$. Каждый вектор $x$, удовлетворяющий этому условию, называется циклическим вектором представления $\pi$. Спектральная кратность представления определяется естественным образом и обозначается через $\mu(\pi)$ (подробнее об этом см. в [1] и [4]). Очевидно, что $\mu(L)=1$, если в алгебре $\mathscr{A}$ имеется единица.

Спектральная кратность - важный инвариант операторов, играющий ключевую роль в теории операторов и ее приложениях. Разумеется, понятие циклического множества связано с общей проблемой инвариантных подпространств, поскольку у оператора $A \in \mathscr{B}(X)$ нет нетривиальных инвариантных подпространств тогда и только тогда, когда всякий вектор $x \in X \backslash\{0\}$ является циклическим для $A$. Циклические векторы используются также в теории приближений полиномами с весом. (Более подробное изложение см., например, в [2]-[6].)

Если даны два представления, $\pi_{1}: \mathscr{A} \rightarrow \mathscr{B}\left(X_{1}\right)$ и $\pi_{2}: \mathscr{A} \rightarrow \mathscr{B}\left(X_{2}\right)$, алгебры $\mathscr{A}$, то их прямая сумма $\pi=\pi_{1} \oplus \pi_{2}: \mathscr{A} \rightarrow \mathscr{B}\left(X_{1} \oplus X_{2}\right)$ определяется равенством $\pi(a)=\pi_{1}(a) \oplus \pi_{2}(a)$. Известно, что

$$
\max \left\{\mu\left(\pi_{1}\right), \mu\left(\pi_{2}\right)\right\} \leqslant \mu\left(\pi_{1} \oplus \pi_{2}\right) \leqslant \mu\left(\pi_{1}\right)+\mu\left(\pi_{2}\right) .
$$

Возникает естественный вопрос: когда $\max \left\{\mu\left(\pi_{1}\right), \mu\left(\pi_{2}\right)\right\}=\mu\left(\pi_{1} \oplus \pi_{2}\right)$ ? Когда, с другой стороны,

$$
\mu\left(\pi_{1} \oplus \pi_{2}\right)=\mu\left(\pi_{1}\right)+\mu\left(\pi_{2}\right) ?
$$

В настоящей статье мы докажем формулу сложения (2) при некоторых дополнительных условиях. Наши рассуждения основываются на известном результате Лина [7] для банаховых алгебр.

2. Основной результат. Пусть $\mathscr{A}$ - коммутативная банахова алгебра с единицей $e$, а $\mathscr{M}=\mathscr{M}(\mathscr{A})$ - пространство ее максимальных идеалов. В статье [7] Лин рассмотрел задачу дополнения строки $a=\left(a_{1}, \ldots, a_{n}\right)$ из элементов $a_{i} \in \mathscr{A}, i=1, \ldots, n$, до $(n \times n)$-матрицы $\left(a_{i j}\right)_{i, j=1}^{n}\left(a_{1 j}=a_{j}, j=1, \ldots, n\right)$ с 
элементами из $\mathscr{A}$, которая обратима в алгебре матриц над $\mathscr{A}$. Если такая матрица существует, то строка $a=\left(a_{1}, \ldots, a_{n}\right)$ называется дополняемой. Очевидное необходимое условие дополняемости строки состоит в существовании таких элементов $b_{i} \in \mathscr{A}, i=1, \ldots, n$, для которых

$$
\sum_{i=1}^{n} a_{i} b_{i}=e
$$

Иными словами, множество $\left\{a_{1}, \ldots, a_{n}\right\}$ не содержится ни в каком собственном идеале алгебры $\mathscr{A}$. Будем говорить, что алгебра $\mathscr{A}$ удовлетворяет условию Лина, если из условия (3) следует, что строка $a=\left(a_{1}, \ldots, a_{n}\right)$ дополняема; Лин доказал [7], что это условие выполнено для алгебр $\mathscr{A}$ со стягиваемым пространством максимальных идеалов $\mathscr{M}=\mathscr{M}(\mathscr{A})$ и для некоторых других алгебр.

Основным результатом настоящей статьи является следующая теорема.

Теорема 1. Пусть $\mathscr{A}$ - коммутативная банахова алгебра с единищей, удовлетворяющая условию Лина, а $L$ - ее регулярное представление. Тогда $\mu(L \oplus$ $\tau)=1+\mu(\tau)$ для любого представления $\tau$ алгебры $\mathscr{A}$.

Сначала мы докажем следующую ключевую лемму.

Лемма 2. Пусть $\pi: \mathscr{A} \rightarrow \mathscr{B}\left(X_{1}\right)$ u $\tau: \mathscr{A} \rightarrow \mathscr{B}\left(X_{2}\right)$ - представления алгебры $\mathscr{A}$ (не обязательно коммутативной), и пусть $\left\{\xi_{1} \oplus \eta_{1}, \ldots, \xi_{n} \oplus \eta_{n}\right\}-$ ииклическое множество представления $\pi \oplus \tau$. Если существует сплетающий onepamop $W \in \mathscr{B}\left(X_{1}, X_{2}\right)($ m.е. $W \pi(b)=\tau(b) W$ для всех $b \in \mathscr{A})$, для которого $W \xi_{1}=\eta_{1}$, mo $\mu(\tau)<n$.

Доказательство. Пусть $\eta \in X_{2}$ - произвольный вектор. Тогда существуют элементы $b_{j, i} \in \mathscr{A}, i=1, \ldots, n$, для которых $\lim _{j} \sum_{i=1}^{n}\left(\pi\left(b_{j, i}\right) \oplus \tau\left(b_{j, i}\right)\right)\left(\xi_{i} \oplus \eta_{i}\right)=$ $0 \oplus \eta$ в пространстве $X_{1} \oplus X_{2}$, т. е. $\lim _{j}\left(\sum_{i=1}^{n} \pi\left(b_{j, i}\right) \xi_{i} \oplus \sum_{i=1}^{n} \tau\left(b_{j, i}\right) \eta_{i}\right)=0 \oplus \eta$. Отсюда следует, что

$$
\begin{aligned}
& \lim _{j} \sum_{i=1}^{n} \pi\left(b_{j, i}\right) \xi_{i}=0 \quad \text { в } X_{1}, \\
& \lim _{j} \sum_{i=1}^{n} \tau\left(b_{j, i}\right) \eta_{i}=\eta \quad \text { в } X_{2} .
\end{aligned}
$$

Используя свойства оператора $W$, из равенства (4) получаем, что

$$
\lim _{j} \sum_{i=1}^{n} W \pi\left(b_{j, i}\right) \xi_{i}=\lim _{j} \sum_{i=1}^{n} \tau\left(b_{j, i}\right) W \xi_{i}=0
$$

в пространстве $X_{2}$. Введем обозначение $\epsilon_{j}=\tau\left(b_{j, 1}\right) \eta_{1}+\sum_{i=2}^{n} \tau\left(b_{j, i}\right) W \xi_{i}$. Очевидно, что $\lim _{j} \epsilon_{j}=0$ в $X_{2}$ и $\tau\left(b_{j, 1}\right) \eta_{1}=\epsilon_{j}-\sum_{i=2}^{n} \tau\left(b_{j, i}\right) W \xi_{i}$. Подставляя это выражение в формулу (5), получаем равенство $\lim _{j}\left(\epsilon_{j}+\sum_{i=2}^{n} \tau\left(b_{j, i}\right)\left(\eta_{i}-\right.\right.$ $\left.\left.W \xi_{i}\right)\right)=\eta$, или $\lim _{j} \sum_{i=2}^{n} \tau\left(b_{j, i}\right)\left(\eta_{i}-W \xi_{i}\right)=\eta$, откуда заключаем, что множество $\left\{\eta_{2}-W \xi_{2}, \ldots, \eta_{n}-W \xi_{n}\right\}$ является циклическим для представления $\tau$, т. е. $\mu(\tau)<n$, что и требовалось. Лемма 2 доказана.

Доказательство теоремы 1. Если $\mu(\tau)=\infty$, то благодаря неравенствам (1) утверждение теоремы очевидно, так что мы будем считать в дальнейшем, что $\mu(\tau)=n<\infty$. 
Заметим, что любой набор векторов в пространстве представления может быть переведен в другой набор при помощи матрицы с элементами из $\mathscr{A}$. Если эта матрица обратима, то циклическое множество переходит в циклическое. Такие множества мы будем называть эквивалентными.

Для доказательства теоремы теперь заметим, что если $\mu(L \oplus \tau)=\mu(\tau)=n$, а множество $\left\{a_{1} \oplus \eta_{1}, \ldots, a_{n} \oplus \eta_{n}\right\}$ является циклическим для $L \oplus \tau$, то (благодаря условию Лина), подействовав на это множество подходящей обратимой матрицей, мы получим эквивалентное множество, в котором $a_{1}=e$. Определим теперь оператор $W: \mathscr{A} \rightarrow X$, положив $W b=\tau(b) \eta_{1}$. Легко видеть, что это сплетающий оператор и что $W e=\eta_{1}$. В самом деле,

$$
W L(a) b=W L_{a} b=W a b=\tau(a b) \eta_{1}=\tau(a) \tau(b) \eta_{1}=\tau(a) W b
$$

при всех $a, b \in \mathscr{A}$, так что $W e=\tau(e) \eta_{1}=\eta_{1}$. Остается сослаться на лемму 2 . Теорема 1 доказана.

Из теоремы 1 вытекают следующие результаты.

Пример 3. Пусть $W_{A}\left(\mathbb{D}^{n}\right)-$ винерова алгебра на полидиске $\mathbb{D}^{n}=\mathbb{D} \times \cdots \times \mathbb{D}$,

$$
W_{A}\left(\mathbb{D}^{n}\right)=\left\{f: f\left(z_{1}, \ldots, z_{n}\right)=\sum_{|\alpha| \geqslant 0} c_{\alpha} z^{\alpha}, \alpha=\left(\alpha_{1}, \ldots, \alpha_{n}\right), \sum_{|\alpha| \geqslant 0}\left|c_{\alpha}\right|<+\infty\right\},
$$

и пусть $\mathscr{T}=\left\{T_{1}, \ldots, T_{n}\right\}$, где $T_{i} f=z_{i} f, f \in W_{A}\left(\mathbb{D}^{n}\right)$. Тогда $\mu(\mathscr{T} \oplus \mathscr{U})=$ $\mu(\mathscr{T})+\mu(\mathscr{U})=1+\mu(\mathscr{U})$ для любого семейства $\mathscr{U}=\left\{U_{1}, \ldots, U_{n}\right\}$ попарно коммутирующих сжимающих операторов $U_{i}$ на банаховом пространстве $X$.

Пример 4. Пусть дана алгебра

$$
C_{A}\left(\mathbb{D}^{n}\right)=\left\{f=\sum_{|\alpha| \geqslant 0} c_{\alpha} z^{\alpha}: f \in \operatorname{Hol}\left(\mathbb{D}^{n}\right) \cap C\left(\overline{\mathbb{D}}^{n}\right)\right\}
$$

аналитических функций на полидиске $\mathbb{D}^{n}$, и пусть $\mathscr{T}=\left\{T_{1}, \ldots, T_{n}\right\}$, где $T_{i} f=$ $z_{i} f, f \in C_{A}\left(\mathbb{D}^{n}\right)$. Тогда $\mu(\mathscr{T} \oplus \mathscr{U})=\mu(\mathscr{T})+\mu(\mathscr{U})=1+\mu(\mathscr{U})$ для любого семейства $\mathscr{U}=\left\{U_{1}, \ldots, U_{n}\right\}$ попарно коммутирующих операторов $U_{i}$, удовлетворяющих условию

$$
\left\|p\left(U_{1}, \ldots, U_{n}\right)\right\| \leqslant c\|p\|_{C_{A}\left(\mathbb{D}^{n}\right)},
$$

где $c>0$ - некоторая константа, а $p-$ произвольный полином.

Автор благодарен рецензенту за конструктивные замечания.

\section{ЛитератУРА}

[1] А. А. Кириллов, Элементы теории представлений, Наука, М., 1972. [2] N. Dunford, L. Schwartz, Linear operators, Part I: General theory, Springer-Verlag, New York, 1958. [3] H. Radjavi, P. Rosenthal, Invariant subspaces, Springer-Verlag, Berlin-New York, 1973. [4] Н. К. Никольский, Инвариантные подпространства в теории операторов и теории функиий, Итоги науки и техники, Математический анализ, т. 12, 1974. [5] Н. К. Никольский, Лекции об операторе сдвига, Наука, М., 1980. [6] B. SzökefalviNagy, C. Foias, Harmonic analysis of operators on Hilbert space, North-Holland, Amsterdam, 1970. [7] В. Я. Лин, Функц. анализ и его прил., 7:2 (1973), 43-51. 